\title{
Affective judgments by patients with Parkinson's disease or chronic progressive multiple sclerosis
}

\author{
WILLIAM W. BEATTY \\ The Neuropsychiatric Institute, Fargo, North Dakota \\ and North Dakota State University, Fargo, North Dakota \\ DONALD E. GOODKIN \\ Fargo Clinic-MeritCare, Fargo, North Dakota \\ and University of North Dakota School of Medicine, Fargo, North Dakota \\ WILLIAM S. WEIR and R. DENNIS STATON \\ University of North Dakota School of Medicine, Fargo, North Dakota \\ and Fargo Veterans Administration Medical Center, Fargo, North Dakota \\ NANCY MONSON \\ The Neuropsychiatric Institute, Fargo, North Dakota \\ and \\ PATRICIA A. BEATTY \\ North Dakota State University, Fargo, North Dakota
}

\begin{abstract}
Both patients with Parkinson's disease or chronic progressive multiple sclerosis and neurologically normal controls judged the emotional expression of faces from a standardized set of photographs. Both groups of patients were impaired on the affective judgment task and on Benton's Facial Recognition Test. Multiple regression analysis showed that performance on the facial recognition test accounted for $44 \%$ of the variance in performance on the affective judgment task, whereas scores on the Beck Depression Inventory did not predict the accuracy of affective judgments. Inaccurate judgments of affect by patients with Parkinson's disease or multiple sclerosis appear to have been secondary to their impaired ability to discriminate details of facial stimuli.
\end{abstract}

Feinberg, Rifkin, Schaffer, and Walker (1986) have reported that patients with major depressive disorders were impaired in labeling emotional expressions of faces, but that they performed normally on tests of facial discrimination that did not require assigning emotional labels to faces. Depression is reported to be common among patients with Parkinson's disease (PD) (Dakof \& Mendelsohn, 1986; Gotham, Brown, \& Marsden, 1986; Mayeaux, Stern, Rosen, \& Leventhal, 1981) or multiple sclerosis (MS) (Devins \& Seland, 1987; Whitlock \& Siskind, 1980), but the processing of emotional stimuli by these patients has received little study. In one investigation, Scott, Caird, and Williams (1984) asked PD patients and controls to match the emotional expressions conveyed in brief spoken passages to appropriate facial expressions in a set of cartoons. The PD patients were impaired on this task, but it is not clear whether this impairment arose because they

This research was supported in part by a grant from the University of North Dakota School of Medicine. We thank Charles Anderson, Richard Bailly, Randall Bjork, Ryan Harrington, and Robert Ivers for allowing us to test their patients, and Jonathan Uecker, who assisted in the testing. Reprint requests may be addressed to William W. Beatty, Department of Psychology, North Dakota State University, Fargo, ND 58105 . had difficulty judging the prosodic content of speech, the emotional expressions of the faces, or both.

In the present study, we examined the ability of PD and MS patients to make judgments of affect using the facial stimuli developed by Ekman and Friesen (1976). Of particular interest was the relationship between judgment of affect and the levels of depression reported by these patients. Since both MS and PD patients may experience difficulties in visual perception (Boller et al., 1984; Heaton, Nelson, Thompson, Burks, \& Franklin, 1985), all subjects also received the Benton Facial Recognition Test, a measure of facial discrimination of nonemotional stimuli.

\section{METHOD}

\section{Subjects}

Twenty-one patients who met diagnostic criteria for chronic progressive, clinically definite MS (Poser et al., 1983) were recruited from the patient population of the North Dakota Multiple Sclerosis Clinic. Chronic progression was defined as observed deterioration in neurological status over a minimum of 6 months, as measured by a 0.5 deterioration on the Expanded Disability Status Scale (EDSS) (Kurtzke, 1983) or a one-grade worsening on the Ambulation Index (AI) (Hauser et al., 1983). Exacerbations (development of one or more new signs or worsening of existing signs when the patient's neurological status had been stable 
or had improved within the last month) were not evident in this group of patients during the study period. The patients averaged 52.0 years of age, 14.0 years of education, 18.4 years of disease since diagnosis, 6.6 on the EDSS, and 6.1 on the AI. At the time of testing, 5 patients were receiving muscle relaxants, 5 were taking oxybutynin for bladder control, and 2 were receiving a tricyclic antidepressant. No patient was receiving ACTH or steroids.

Forty-three patients with idiopathic PD were referred by area neurologists. The patients included in the PD group had to have shown at least two of the following signs: masked facies, tremor at rest, cogwheel rigidity, bradykinesia, or disturbance of postural reflexes. They also had to have shown improvement in their neurological symptoms following treatment with dopaminergic medications if such treatment had been tried. (All but one of the patients had received a trial with dopaminergic medication.) Patients from Stages 1-4 on the Hoehn and Yahr (1967) scale were accepted into the study; most of the patients were in Stage 2 or 3. No PD patient had undergone thalamotomy or any other neurosurgical procedure. The patients averaged 65.8 years of age, 12.5 years of education, and 4.7 years of disease since diagnosis. At the time of testing, all but two of the patients were taking one or more dopaminergic medications (1-dopa in combination with carbidopa, bromocriptine, or amantidine). Six patients were also receiving anticholinergic therapy (trihexiphenydyl or benztropine), 4 were taking the antihistamine diphenhydramine, and 3 were taking a tricyclic antidepressant.

Since the PD patients were older and less well educated than the MS patients, two groups of neurologically normal control subjects were recruited from the community and paid for their participation. The MS controls $(N=19)$ averaged 51.1 years of age and 14.4 years of education. The PD controls $(N=27)$ averaged 64.6 years of age and 12.7 years of education. All subjects gave written informed consent after a thorough explanation of the procedures. Patients with a history of major psychiatric illness, drug or alcohol abuse, head injury, or CNS diseases other than MS or PD were excluded.

\section{Materials Procedures}

All the subjects were first given the Mini-Mental State Examination (MMSE) (Folstein, Folstein, \& McHugh, 1975) and the Beck Depression Inventory (BDI) (Beck, Ward, Mendelson, Mock, \& Erbaugh, 1961). Next, the short form of the Benton Facial Recognition Test (BFRT) (Benton, Hamsher, Varney, \& Spreen, 1983) was administered. Finally, the subjects judged the 110 faces representing standardized poses of fundamental human emotions developed by Ekman and Friesen (1976). These stimuli were presented as black-and-white photographs mounted on index cards. The subjects were told to select the emotion (happy, sad, angry, afraid, disgusted, surprised, or neutral) that best described the emotional state represented by each photograph. An index card listing the 7 alternative choices (see above) remained in the subjects' view throughout the testing. No time limits were imposed on the subjects' responses for any measure.

\section{RESULTS}

As shown in Table 1, both the MS and the PD patients scored higher on the BDI (i.e., they were more depressed) than did their respective control groups $[F(1,68)=22.63$, $p<.001$ for PD patients; $F(1,38)=7.61, p<.01$ for
MS patients]. For the PD patients, scores on the BDI ranged from 0 to $22 ; 19$ of the 43 PD patients scored above 9. For the MS patients, scores ranged from 0 to $24 ; 6$ of the 21 patients scored above 9 .

Both PD $[F(1,68)=17.48, p<.001]$ and MS $[F(1,38)$ $=5.13, p<.05]$ patients scored below their controls on the MMSE. Eighteen of the PD patients scored below 28 , which was the lower limit of the range for both control groups, but only 3 of the PD patients scored below 24 . Five of the MS patients scored below 28, but only 1 MS patient scored below 24. Scores on the BDI and MMSE were not significantly correlated for either patient group $(r s<.17)$.

Compared to their respective controls, both $P D$ patients $[F(1,68)=8.24, p<.01]$ and MS patients $[F(1,38)=$ $15.29, p<.001]$ were less accurate in judging emotional expressions conveyed by the photographs depicting fundamental affective states. Both patient groups were also less accurate in discriminating neutral faces, as is evidenced by their significantly lower scores on the BFRT $[F(1,68)=20.86, p<.001$ for PD patients; $F(1,38)=$ $14.14, p<.001$ for MS patients].

Subsequent analyses of the distribution of errors by the patients and controls indicated that the patients were equally impaired in judging stimuli depicting each of the seven emotional states, and that they showed no particular tendency to confuse faces (e.g., happy and sad) expressing emotions of opposite polarity.

To examine the relationship among overall accuracy of affective judgments and potential predictor variables, product-moment correlations were computed separately for each of the four groups. These analyses, shown in Table 2, revealed positive correlations between scores on the BFRT and those on the affective judgment task, which were statistically significant for each of the four groups. In contrast, the relationships between scores on the BDI and on the affective judgment measure, while in the predicted direction, did not approach statistical significance. Correlations of affective judgments with age, education, or MMSE scores were significant for some groups but not for others, most likely reflecting idiosyncratic patterns of correlations of age, education, and MMSE scores with performance on the BFRT.

The correlational analyses described above suggested that the accuracy of subjects' affective judgments of facial stimuli could be predicted from knowledge of their performance on the BFRT, and that scores on the BDI contributed little to predicting accuracy of judging emo-

Table 1

Mean Performance (with Standard Deviations) of Patients with Parkinson's Disease or Multiple Sclerosis and Controls

\begin{tabular}{|c|c|c|c|c|c|c|c|c|}
\hline & \multicolumn{2}{|c|}{ PD } & \multicolumn{2}{|c|}{ PD Controls } & \multicolumn{2}{|c|}{ MS } & \multicolumn{2}{|c|}{ MS Controls } \\
\hline & $M$ & $S D$ & $M$ & $S D$ & $M$ & $S D$ & $M$ & $S D$ \\
\hline Mini-Mental State Examination & 27.3 & 2.2 & 29.2 & 0.9 & 28.2 & 2.4 & 29.5 & 0.8 \\
\hline Beck Depression Inventory & 9.8 & 5.5 & 3.7 & 4.6 & 7.3 & 6.6 & 2.4 & 4.3 \\
\hline Affective Judgment/110 & 77.8 & 14.8 & 87.1 & 10.1 & 75.0 & 13.4 & 90.4 & 11.2 \\
\hline Benton Facial Recognition Test/27 & 19.1 & 3.0 & 22.4 & 2.8 & 19.1 & 3.6 & 23.2 & 3.3 \\
\hline
\end{tabular}


Table 2

Product-Moment Correlations of Overall Affective Judgment Scores with Other Measures, for Patients with Parkinson's Disease or Multiple Sclerosis and Controls

\begin{tabular}{lcccc}
\hline & PD & PD Controls & MS & MS Controls \\
\hline Age & -.25 & -.33 & .15 & $-.58 \dagger$ \\
Years of Education & $.38^{*}$ & -.33 & $.45^{*}$ & .18 \\
Beck Depression Inventory & -.17 & -.20 & -.16 & -.35 \\
Mini-Mental State Examination & $.49 \dagger$ & -.08 & .40 & $.52^{*}$ \\
Benton Facial Recognition Test & $.57 \dagger$ & $.57 \dagger$ & $.49^{*}$ & $.75 \dagger$ \\
\hline
\end{tabular}

${ }^{*} p<.05 \quad \dagger p<.01$.

tions conveyed by facial expressions. This hypothesis was tested by using stepwise multiple regression to predict the overall accuracy of affective judgments (i.e., the total number correct). The analysis, which included all four groups, indicated that performance on the BFRT accounted for $43.6 \%$ of the variance in performance on the affective judgment task. Scores on the MMSE increased the accuracy of prediction significantly, accounting for an additional $3.0 \%$ of the variance. Age, education, BDI scores, and group membership did not contribute significantly to predicting the accuracy of affective judgments. Two separate regression analyses, one including MS patients and their controls, the other including PD patients and their controls, revealed essentially the same results.

Subsequently, we have studied 42 patients with the relapsing remitting type of MS. Relative to age-, education-, and gender-matched controls, these patients were only slightly impaired on the BFRT, and, as a group, they performed normally on the affective judgment task (Mean $=91.5$ correct). The two patients whose BFRT scores placed them in the clinically impaired range scored 68 and 71 , respectively, on the affective judgment task.

\section{DISCUSSION}

The present findings demonstrate that patients with chronic progressive MS or PD are impaired in labeling emotional expressions from photographs of facial stimuli. These impairments were not restricted to particular emotional states or to stimuli of a positive or negative valence, and the distribution of the errors made by the patients differed only slightly from that of the controls. In particular, neither the MS nor the PD patients showed any marked tendency to confuse positive and negative emotions. These observations indicate that the central mechanisms that allow accurate labeling of affective states remain largely intact in MS and PD, but the accuracy of the operation of these mechanisms is defective.

The MS and PD patients were also impaired on the BFRT, a task that requires discrimination of facial features, but does not require emotional labeling. Since discriminating facial features is presumably an essential prerequisite for accurate labeling of emotions from photographs, it seems likely that the patients' difficulties in judging affect are secondary to their deficits in discriminating facial stimuli. Our data cannot establish this point firmly, but the consistent pattern of positive correlations between scores on the BFRT and those on judgments of affect, which were observed in both patient groups and among controls, suggests that impairments in the judgment of emotional expression from facial stimuli can be predicted from performance on the BFRT-a brief, easily administered, and well-standardized test.

The pathophysiological basis of these visuoperceptual deficits is uncertain, but disturbances in optic pathways are likely to be important. Abnormal visual evoked potentials are commonly observed in patients with MS (Halliday, McDonald, \& Mushin, 1973), and they also may occur in patients with PD (Bodis-Wollner \& Yahr, 1978; Regan \& Neima, 1984). Ebers (1986) has estimated that about $90 \%$ of MS patients suffer optic nerve damage while Bodis-Wollner, Yahr, Mylin, and Thornton (1982) have suggested that dopamine deficiency in the retina might result in visual dysfunction in PD. Regan and Maxner (1987) have described several similarities in the pattern of visual loss in PD and in MS, which may arise from loss of inputs to the striate cortex. Generalized cerebral hypometabolism, which has been reported to occur in patients with MS (Brooks et al., 1984) and PD (Kuhl, Metter, Riege, \& Markham, 1984), may also have contributed to the visuoperceptual difficulties exhibited by the clinical groups we studied.

On the basis of an earlier report (Feinberg et al., 1986) that depressed patients exhibited deficits in judging affect from facial stimuli, we had predicted that patients with MS or PD who had high scores on the BDI would have particular difficulty in accurately labeling the emotional states conveyed by facial stimuli. However, BDI scores were not significantly related to the accuracy of making affective judgments in this study.

Several factors may explain this apparent discrepancy. Feinberg et al. (1986) studied hospitalized depressed patients, while only a small number of our MS and PD patients were undergoing (outpatient) treatment for depression. None of our patients was regularly seeing a psychiatrist. Further, although 25 of our patients attained BDI scores that placed them in the depressed range (i.e., above 9), the extent of their depression may have been overestimated, because many of the items on the BDI concern physical symptoms that may occur in PD or MS. Moreover, fewer than $50 \%$ of the patients within each clinical group had BDI scores within the depressed range. It is reasonable, therefore, to assume that our clinical groups were not as severely depressed as the patients studied by Feinberg et al. (1986). This may explain why we observed little relationship between BDI scores and judgments of affect.

Feinberg et al. (1986) presented the facial stimuli tachistoscopically and allowed only $0.5 \mathrm{sec}$ of exposure, whereas in the present study, exposure was self-paced: the subjects could view the stimuli for as long as they wished. This procedural difference might also be important, since the self-paced format used in the present study should have been less effortful than the time-limited procedure used by Feinberg et al. (1986) Research by Weingartner and his colleagues (Cohen, Weingartner, Smallberg, Pickar, \& Murphy, 1982; Roy-Byrne, Weingartner, Bierer, Thompson, \& Post, 1986; Weingartner, Cohen, Murphy, Martello, \& Gerdt, 1981) indicates that depressed subjects are likely to perform poorly when the cognitive demands of a task place a premium on effortful processing.

Whether or not degree of depression colors judgment of affect, the impairments demonstrated in the present study imply that some patients with PD or MS probably experience difficulties in trying to make accurate visual affective judgments under conditions of real life, when spontaneous expressions occur briefly and in the context of multiple sources of information input. Since in real life facial expressions provide only one source of information upon which affective judgments can be based, we cannot conclude that such patients experience functional impairments. Patients might utilize linguistic inputs or prosodic information from nonvisual sources to compensate for their difficulties in making visual affective judgments. The effectiveness of such compensatory strategies may be limited, however, since previous research (Scott et al., 1984) indicates that patients with PD have difficulty match- 
ing the emotional information conveyed by facial and vocal stimuli. Naturalistic study of the interactions of PD and MS patients with others in their home environments could establish whether or not these interactions are adversely compromised by impaired capacity to recognize and respond appropriately to the varying moods of other persons.

\section{REFERENCES}

Beck, A. T., Ward, C. H., Mendelson, M., Mock, J., \& Erbaugh, J. (1961). An inventory for measuring depressions. Archives of General Psychiatry, 4, 561-571.

Benton, A. L., Hamsher, K. de S., Varney, N. R., \& Spreen, O. (1983). Contributions to neuropsychological assessment. New York: Oxford University Press.

Bodis-Wollner, I., \& YAHR, M. D. (1978). Measurements of visual evoked potentials in Parkinson's disease. Brain, 101, 661-671.

Bodis-Wollner, I., YAHR, M. D., MyLin, L., \& ThORNTON, J. (1982). Dopaminergic deficiency and delayed visual evoked potentials in humans. Annals of Neurology, 11, 478-483.

Boller, F., Passafiume, D., Keefe, N. C., Rogers, K., Morrow, L., \& KIM, Y. (1984). Visuospatial impairment in Parkinson's disease: Role of perceptual and motor factors. Archives of Neurology, 41, 485-490.

Brooks, D. J., Leenders, K. L., Head, G., Marshall, J., LegG, N. J., \& JONES, T. (1984). Studies on regional cerebral oxygen utilisation and cognitive function in multiple sclerosis. Journal of Neurology, Neurosurgery \& Psychiatry, 47, 1182-1191.

Cohen, R. M., Weingartner, H., Smallberg, S. A., Pickar, D., \& MURPHY, D. L. (1982). Effort and cognition in depression. Ar chives of General Psychiatry, 39, 593-597.

Dakof, G. A., \& Mendelsohn, G. A. (1986). Parkinson's disease: The psychological aspects of a chronic illness. Psychological Bulletin, 99, 375-387.

Devins, G. M., \& Seland, T. P. (1987). Emotional impact of multiple sclerosis: Recent findings and suggestions for future research. Psychological Bulletin, 101, 363-375.

EBERs, G. C. (1986). Multiple sclerosis and other demyelinating diseases. In A. D. Asbury, G. M. McKhann, and W. I. McDonald (Eds.), Diseases of the nervous system: Clinical neurobiology. (Vol. 2, pp. 1268-1281). Philadelphia, PA: W. B. Saunders.

Ekman, P., \& Friesen, W. (1976). Pictures of facial affect. Palo Alto, CA: Consulting Psychologists Press.

Feinberg, T. E., Rifkin, A., Schaffer, C., \& Walker, E. (1986). Facial discrimination and emotional recognition in schizophrenia and affective disorders. Archives of General Psychiatry, 43, 276-279.

Folstein, M. F., Folstein, S. E., \& McHugh, P. R. (1975). “MiniMental State": A practical method for grading the cognitive state of patients for the clinician. Journal of Psychiatric Research, 12, 189-198. Gotham, A. M., Brown, R. G., \& MARSDEn, C. M. (1986). Depres- sion in Parkinson's disease: Quantitative and qualitative analysis. Journal of Neurology, Neurosurgery \& Psychiatry, 49, 381-389.

Halliday, A. M., McDonald, W. I., \& Mushin, J. (1973). Visual evoked potentials in the diagnosis of multiple sclerosis. British Medical Journal, 4, 661-664.

Hauser, S. L., Dawson, D. M., Lehrich, J. B., Beal, M. F., KeVy, S. V., Propper, R. D., Mills, J. A., \& Weiner, H. L. (1983). Intensive immunosuppression in progressive multiple sclerosis. New England Journal of Medicine, 308, 173-180.

heaton, R. K., Nelson, L. M., Thompson, D. S., Burks, J. S., \& FrankLIN, G. M. (1985). Neuropsychological findings in relapsingremitting and chronic-progressive multiple sclerosis. Journal of Consulting Clinical Psychology, 53, 103-110.

HoEHN, M. M., \& YAHR, M. D. (1967). Parkinsonism: Onset, progression, and mortality. Neurology, 17, 427-442.

Kuhl, D. E., Metter, E. J., Riege, W. H., \& Markham, C. H. (1984). Patterns of cerebral glucose utilization in Parkinson's disease and Huntington's disease. Annals of Neurology, 15, 119-125.

KURTZKE, J. F. (1983). Rating neurological impairment in multiple sclerosis: An expanded disability status scale (EDSS). Neurology, 33, 1444-1452.

Mayeaux, R., STern, Y., Rosen, J., \& LeVenthal, J. (1981). Depression, intellectual impairment, and Parkinson disease. Neurology, 31, 645-650.

Poser, C. M., Paty, D. W., Scheinberg, L., McDonald, W. I., Davis, F. A., Ebers, G. C., Johnson, K. P., Sibley, W. A., Silberberg, D. H., \& Tourtellotte, W. W. (1983). New diagnostic criteria for multiple sclerosis: Guidelines for research protocols. Annals of Neurology, 13, 227-231.

Regan, D., \& MaXner, C. (1987). Orientation-selective visual loss in patients with Parkinson's disease. Brain, 110, 415-432.

Regan, D., \& NeIma, D. (1984). Visual fatigue and visual evoked potentials in multiple sclerosis, glaucoma, ocular hypertension and Parkinson's disease. Journal of Neurology, Neurosurgery \& Psychiatry, 47, 673-678.

Roy-Byrne, P. P., Weingartner, H., Bierer, L. M., Thompson, K., \& Post, R. M. (1986). Effortful and automatic cognitive processes in depression. Archives of General Psychiatry, 43, 265-267.

Scott, S., Caird, F. I., \& William, S. B. (1984). Evidence for an apparent sensory speech disorder in Parkinson's disease. Journal of Neurology, Neurosurgery \& Psychiatry, 47, 840-843.

Weingartner, H., Cohen, R. M., Murphy, D. L., Martello, J., \& GERDT, C. (1981). Cognitive processes in depression. Archives of General Psychiatry, 38, 42-47.

Whitlock, F. A., \& Siskind, M. M. (1980). Depression as a major symptom of multiple sclerosis. Journal of Neurology, Neurosurgery \& Psychiatry, 43, 861-865.

(Manuscript received December 28, 1988.) 